\title{
PARAMETRICALLY DRIVEN OSCILLATORS WITH ADDED NOISE: HEATING AND THERMAL SQUEEZING
}

\author{
ADRIANO A. BATISTA* \\ * Departamento de Física, Universidade Federal de Campina Grande, \\ Campina Grande-PB 58109-970, Brazil \\ Email: adriano@df.ufcg.edu.br
}

\begin{abstract}
Here we report a theoretical model based on Green's functions, Floquet theory, and averaging techniques up to second order that describes the dynamics of parametrically driven oscillators with added thermal noise. Quantitative estimates for heating and quadrature thermal noise squeezing near and below the transition line of the first parametric instability zone of the oscillator are given. Furthermore, we give an intuitive explanation as to why heating and thermal squeezing occur. Finally, we validate our analytical estimates of thermal fluctuations by verifying them numerically. Very good agreement is achieved between the two approaches.
\end{abstract}

Keywords - Parametric oscillator, thermal noise squeezing, stochastic dynamics.

Resumo - Aqui reportamos um modelo teórico baseado em funções de Green, teoria de Floquet e técnicas de averaging até segunda ordem que descrevem a dinâmica de osciladores parametricamente forçados com ruído térmico adicionado. Estimativas quantitativas para o aquecimento e a compressão em quadratura do ruído térmico próximo ao limiar da primeira zona de instabilidade do oscilador são dados. Além disso, explicamos intuitivamente por que a compressão térmica e o aquecimento ocorrem. Finalmente, validamos nossas estimativas analíticas das flutuações térmicas verificando-as numericamente. Obtemos uma ótima concordância entre as duas abordagens.

Palavras-chave — Oscilador paramétrico, compressão térmica de ruído, dinâmica estocástica

\section{Introduction}

Motivated by the seminal experimental work of Rugar and Grütter (Rugar and Grütter, 1991) on thermal squeezing in a parametrically-driven MEMS device, we study a parametric oscillator in the presence of noise with the objective of understanding what are the main causes of thermal squeezing and heating that occur in this system when its parameters are set below the first parametric instability. The dynamical system model studied here may be used, for instance, to describe the time evolution of the fundamental mode of a doubly-clamped beam resonator that is axially loaded, in which case the dynamical variable of our model represents the amount of deflexion at the middle of the beam from the equilibrium position.

In this paper we extend and improve on recently obtained analytical quantitative estimates of the amount of quadrature noise squeezing and heating in a parametrically-driven oscillator (Batista, 2011). Here we use the Green's function approach, previously developed to solve the Langevin equation, aligned with averaging techniques up to second order, to obtain more precise analytical estimates of the thermal fluctuations in the parametrically-driven oscillator with added noise. We further show, using an approximate Floquet theory based on first and second-order averaging approximations, that thermal squeezing and heating are related to the onset of real-valued Floquet multipliers (FMs) with different magnitudes. It is shown that one FM grows while one gets closer in parameter space to the first transition line to instability while the other FM decreases. As a consequence, one gets two different effective dissipation rates, while at the same time the input power due to noise remains constant as the pump amplitude is increased. We show below that these effects account for most thermal squeezing and heating observed. Furthermore, second-order averaging analytical estimates of heating and the amount of squeezing are also provided.

\section{Theory}

\subsection{The parametric oscillator}

The equation for the parametrically-driven oscillator (in dimensionless format) is given by the damped Matthieu's equation

$$
\ddot{x}+\omega_{0}^{2} x=-\gamma \dot{x}+F_{p} \cos (2 \omega t) x,
$$

in which $\gamma$ and $F_{p} \sim O(\varepsilon)$, where $\varepsilon<<1$. Since we want to apply the averaging method (AM) (Verhulst, 1996), (Guckenheimer and Holmes, 1983) to situations in which we have detuning, it is convenient to rewrite Eq. (1) in a more appropriate form with the notation $\Omega=\omega_{0}^{2}-\omega^{2}$, where we also have $\Omega \sim O(\varepsilon)$. With this substitution we obtain $\ddot{x}+\omega^{2} x=-\Omega x-\gamma \dot{x}+F_{p} \cos (2 \omega t) x$. we obtain the fundamental matrix $\Phi(t)$ (also known as the time evolution operator) in first-order averaging approximation 


$$
\begin{aligned}
\Phi(t) & \approx e^{-\gamma t / 2}\left(\begin{array}{cc}
\cos \omega t & \frac{1}{\omega} \sin \omega t \\
-\omega \sin \omega t & \cos \omega t
\end{array}\right) \\
\times & \left(\begin{array}{cc}
\cosh (\kappa t) & -\frac{\beta-\delta}{\omega \kappa} \sinh (\kappa t) \\
-\frac{\omega(\beta+\delta)}{\kappa} \sinh (\kappa t) & \cosh (\kappa t)
\end{array}\right)
\end{aligned}
$$

where $\Phi(0)=I$, in which $I$ is the identity matrix. The shorthand parameters are defined as $\kappa=\sqrt{\beta^{2}-\delta^{2}}, \beta=-F_{p} / 4 \omega$, and $\delta=\Omega / 2 \omega$. From Floquet theory (Verhulst, 1996) we know that the fundamental matrix can be written as $\Phi(t)=P(t) e^{B t}$, where $P(t)$ is a periodic matrix with period $\pi / \omega$ and $B$ is a constant matrix. We also know that $P(0)=I$. The eigenvalues of $\Phi(\pi / \omega)=e^{B \pi / \omega}$ are known as the Floquet multipliers (FMs). In 1st-order approximation The FMs are given by

$$
\lambda_{ \pm}=-e^{-\left(\frac{\gamma}{2} \pm \kappa\right) \pi / \omega} .
$$

We can write the fundamental matrix in secondorder approximation as

$$
\begin{gathered}
\Phi(t) \approx e^{-\gamma t / 2}\left(\begin{array}{cc}
\cos \omega t & \frac{1}{\omega} \sin \omega t \\
-\omega \sin \omega t & \cos \omega t
\end{array}\right) \\
\times\left(\begin{array}{cc}
\cosh (\xi t)+a \frac{\sinh (\xi t)}{\xi} & -\frac{b-c}{\omega \xi} \sinh (\xi t) \\
-\frac{\omega(b+c)}{\xi} \sinh (\xi t) & \cosh (\xi t)-a \frac{\sinh (\xi t)}{\xi}
\end{array}\right),
\end{gathered}
$$

where $\xi=\sqrt{a^{2}+b^{2}-c^{2}}, a=-\frac{\gamma \beta}{2 \omega}, b=\beta-\delta \beta / \omega$, and $c=\delta-\gamma^{2} /(8 \omega)-\delta^{2} /(2 \omega)-\beta^{2} /(4 \omega)$. We find the FMs to be given by

$$
\lambda_{ \pm}=-e^{-(\gamma / 2 \pm \xi) \pi / \omega} .
$$

The corresponding effective dissipation rates are $\gamma_{\text {eff }}=\gamma \pm 2 \xi$.

\subsection{Green's functions}

The equation for the Green's function of the parametrically-driven oscillator is given by

$$
\left[\frac{\partial^{2}}{\partial t^{2}}+\omega_{0}^{2}+\gamma \frac{\partial}{\partial t}-F_{p} \cos (2 \omega t)\right] G\left(t, t^{\prime}\right)=\delta\left(t-t^{\prime}\right) .
$$

Since we are interested in the stable zones of the parametric oscillator, for $t<t^{\prime} G\left(t, t^{\prime}\right)=0$ and by integrating the above equation near $t=t^{\prime}$, we obtain the initial conditions when $t=t^{\prime}+0^{+}$, $G\left(t, t^{\prime}\right)=0$ and $\frac{\partial}{\partial t} G\left(t, t^{\prime}\right)=1.0$.

The Green's function of Eq. (4) may be obtained from the operator $H\left(t, t^{\prime}\right)=\Phi(t) \Phi^{-1}\left(t^{\prime}\right)$. The Green's function is given by $G\left(t, t^{\prime}\right)=H_{12}\left(t, t^{\prime}\right)$

\subsubsection{1st-order averaging approximate Green's function}

Although Eq. (4) may be solved exactly by using Floquet theory (Wiesenfeld and McNamara, 1985), one obtains very complex solutions. Instead, we find fairly simple analytical approximations to the Green's functions and, subsequently, to the statistical averages of fluctuations using the averaging method. We find the approximate Green's function near the onset of instability (when $\kappa$ is real) to be

$$
\begin{aligned}
& G(t, t-\tau) \approx \frac{e^{-\gamma \tau / 2}}{\omega}\{\cosh (\kappa \tau) \sin (\omega \tau) \\
& \left.+\frac{\sinh (\kappa \tau)}{\kappa}[\delta \cos (\omega \tau)-\beta \cos (\omega(2 t-\tau))]\right\},
\end{aligned}
$$

for $\tau>0$ and $G(t, t-\tau)=0$ for $\tau<0$.

\subsubsection{2nd-order averaging approximate Green's function}

The Green's function obtained by second-order averaging is given by

$$
\begin{aligned}
& G(t, t-\tau) \approx \frac{e^{-\gamma \tau / 2}}{\omega}\{\cosh (\xi \tau) \sin (\omega \tau) \\
& \left.+\frac{\sinh (\xi \tau)}{\xi}\left[c \cos (\omega \tau)-\beta^{\prime} \cos \left[\omega(2 t-\tau)-\phi_{0}\right]\right]\right\}
\end{aligned}
$$

for $\tau>0$ and $G(t, t-\tau)=0$ for $\tau<0$, where $\beta^{\prime}=\beta \sqrt{(1-\delta / \omega)^{2}+\gamma^{2} /\left(4 \omega^{2}\right)}, a=\beta^{\prime} \sin \left(\phi_{0}\right)$, $b=\beta^{\prime} \cos \left(\phi_{0}\right)$, and $\phi_{0}=2 \omega t_{0}$. We have to replace $\kappa$ by $\xi, \delta$ by $c, \beta$ by $\beta^{\prime}$ and $t$ by $t-t_{0}$ to obtain the second-order Green's function from the 1st-order approximation. This indicates that a Green's function renormalization is very well feasible if one goes to higher orders of approximation in the averaging method.

\subsection{Thermal fluctuations}

We will now investigate the effect of noise on the parametric oscillator (Batista, 2011). We start by adding noise to Eq. (1) and obtain

$$
\ddot{x}=-\omega_{0}^{2} x-\gamma \dot{x}+F_{p} \cos (2 \omega t) x+R(t),
$$

where $R(t)$ is a random function that satisfies the statistical averages $\langle R(t)\rangle=0$ and $\left\langle R(t) R\left(t^{\prime}\right)\right\rangle=2 T \gamma \delta\left(t-t^{\prime}\right)$, according to the fluctuation-dissipation theorem (Kubo, 1966). Using the Green's function we obtain the solution $x(t)$ of Eq. (7) in the presence of noise $R(t)$

$$
x(t)=x_{h}(t)+\int_{-\infty}^{\infty} d t^{\prime} G\left(t, t^{\prime}\right) R\left(t^{\prime}\right),
$$

where $x_{h}(t)$ is the homogeneous solution, which in the stable zone decays exponentially with time; since we assume the pump has been turned on for a long time, $x_{h}(t)=0$. By statistically averaging the fluctuations of $x(t)$ we obtain

$$
\begin{gathered}
\left\langle x^{2}(t)\right\rangle=\iint_{-\infty}^{\infty} d t^{\prime} d t^{\prime \prime} G\left(t, t^{\prime}\right) G\left(t, t^{\prime \prime}\right)\left\langle R\left(t^{\prime}\right) R\left(t^{\prime \prime}\right)\right\rangle \\
=2 T \gamma \int_{0}^{\infty} d \tau G(t, t-\tau)^{2}
\end{gathered}
$$


where $\tau=t-t^{\prime}$. An estimate of the statistically averaged thermal fluctuations, when $|\beta|>|\delta|$, is given by

$$
\left\langle x^{2}(t)\right\rangle \approx \overline{\left\langle x^{2}(t)\right\rangle}+A_{2 \omega} \cos (2 \omega t)+B_{2 \omega} \sin (2 \omega t)
$$

The dc contribution to fluctuations in the secondorder approximation

$$
\begin{aligned}
\overline{\left\langle x^{2}(t)\right\rangle} \approx & \frac{2 \gamma T}{\omega^{2}} \int_{0}^{\infty} d \tau e^{-\gamma \tau}\{[\cosh (\xi \tau) \sin (\omega \tau) \\
& \left.\left.+c \frac{\sinh (\xi \tau)}{\xi} \cos (\omega \tau)\right]^{2}+\beta^{\prime 2} \frac{\sinh ^{2}(\xi \tau)}{2 \xi^{2}}\right\},
\end{aligned}
$$

Notice that when one gets close to the zone of instability, we obtain a far simpler expression for the average fluctuations. It is given approximately by

$$
\begin{aligned}
\left\langle x^{2}(t)\right\rangle & \approx \frac{2 T}{\omega^{2}}\left[\beta^{2}+\frac{\gamma^{2}}{4}+\delta^{2}\right] \frac{1}{\gamma^{2}-4 \kappa^{2}} \\
& -\frac{4 \beta T}{\omega^{2}\left(\gamma^{2}-4 \kappa^{2}\right)}\left[\delta \cos (2 \omega t)+\frac{\gamma}{2} \sin (2 \omega t)\right] .
\end{aligned}
$$

It is easy to verify that the minimum of the position fluctuation is given by

$$
\sigma_{\min }^{2} \equiv\left\langle x^{2}(t)\right\rangle_{\min } \approx \frac{T}{2 \omega^{2}} \frac{\sqrt{\delta^{2}+\gamma^{2} / 4}-|\beta|}{\sqrt{\delta^{2}+\gamma^{2} / 4}+|\beta|}
$$

and the maximum is given by

$$
\sigma_{\max }^{2} \equiv\left\langle x^{2}(t)\right\rangle_{\max } \approx \frac{T}{2 \omega^{2}} \frac{\sqrt{\delta^{2}+\gamma^{2} / 4}+|\beta|}{\sqrt{\delta^{2}+\gamma^{2} / 4}-|\beta|},
$$

such that

$$
\sigma_{\min } \sigma_{\max } \approx \frac{T}{2 \omega^{2}} .
$$

This is a verification that the classical phenomenon of thermal squeezing indeed occurs near the transition line to instability.

The instantaneous input power due to the additive noise is given by

$$
\begin{aligned}
P_{\text {noise }}(t) & =\dot{x}(t) R(t)=v_{h}(t) R(t) \\
& +\int_{-\infty}^{\infty} d t^{\prime} \frac{\partial}{\partial t} G\left(t, t^{\prime}\right) R\left(t^{\prime}\right) R(t) .
\end{aligned}
$$

Hence, we obtain the statistically averaged noise input power

$$
\begin{aligned}
\left\langle P_{\text {noise }}(t)\right\rangle & =\int_{-\infty}^{t} d t^{\prime} \frac{\partial}{\partial t} G\left(t, t^{\prime}\right)\left\langle R\left(t^{\prime}\right) R(t)\right\rangle \\
& =\gamma T\left[\frac{\partial}{\partial t} G\left(t, t^{\prime}\right)\right]_{t^{\prime}=t^{-}}=\gamma T .(14)
\end{aligned}
$$

More details can be found in Refs. (Batista, 2012)(Batista and R. S. N. Moreira, 2011).

\section{Results and discussion}

When the FMs given by Eq. (3) become real, they branch off in two magnitudes. When this occurs one of the FMs $\left(\lambda_{-}\right)$becomes larger as $F_{p}$ is increased, eventually becoming larger than one, when the system given by Eq. (1) becomes unstable, while the other FM $\left(\lambda_{+}\right)$becomes smaller. From Eq. (3) this implies into two effective dissipation rates, along the stable manifolds of the Poincaré first-return map (with period $\pi / \omega$, i.e. $\Phi(\pi / \omega))$. This phenomenon causes both heating and squeezing of the parametric oscillator with added noise, since the average power input due to thermal noise remains constant as is shown in Eq. (14) and one effective dissipation rate is decreased. It also causes quadrature thermal squeezing since in one direction, the $\lambda_{-}$-stable manifold of $\Phi(\pi / \omega)$ in the phase space of $u$ and $v$, there is less effective dissipation and, consequently, more fluctuations, while in another direction, the $\lambda_{+-}$ stable manifold of $\Phi(\pi / \omega)$ in the phase space of $u$ and $v$, there is more effective dissipation and , consequently, less fluctuations. This imbalance in the effective dissipation rates, we claim, is the main cause of thermal squeezing in the parametric oscillator with added noise. The dependence on pump amplitude of the effective dissipations $\left(\gamma_{\text {eff } \pm}=\gamma \pm 2 \xi\right)$ can be seen on Fig. 2 (secondorder averaging result).

In Fig. 3 we show several squared Green's functions with initial conditions spread out evenly in time during one period of the pump $(\pi / \omega)$. They are vertically spaced only for clarity, since all of their asymptotes are zero. The Green's functions are shown squared because that is the way they contribute to the thermal fluctuations in Eq. (8). One notices that the second-order approximation Green's functions yield a much better approximation to the numerical Green's functions than the first-order approximation. This is specially evidenced the closer one gets to the first transition line to instability, a consequence of the fact that the second-order analytical expression for the transition line is a better approximation than the first-order expression.

In Fig. 4 we show a comparison between time series of the fluctuation $\left\langle x^{2}(t)\right\rangle$ given by Eq. (8) in which the Green's functions are given either by the second-order approximation expression from Eq. (6) or by the numerical Floquet theory Green's functions. We use several different Green's functions with negative detuning $(\omega=$ $0.95)$, in resonance $(\omega=1.0)$ and positive detuning $(\omega=1.05)$ all with the same pump amplitude $F_{p}=0.185$. We observe that the numerical and approximate Green's functions are very similar and that the squeezing amplitude and heating (proportional to the time average of $\left\langle x^{2}(t)\right\rangle$ ) are very dependant on detuning from resonance. 
From Fig. 1 one sees that the off resonance results are slightly below the threshold of the strong heating and squeezing zone.

In Fig. 5 we show a plot of the dc component of the mean-square displacement $\left\langle x^{2}(t)\right\rangle$ (in second-order approximation, as given by Eqs. (11)) over the heat bath temperature in decibel scale. Most of the heating occurs inside the heating zone in which the Floquet multipliers are real.

In Fig. 6 we show level sets in decibels of the dc amplitude of the mean-square displacement $\left\langle x^{2}(t)\right\rangle$ in second-order approximation, as given by Eqs. (11), over the heat bath temperature. One sees that most of the heating occurs inside the heating zone in which the FMs are real.

In Fig. 7we show level sets of the ac amplitude of the mean-square displacement $\left\langle x^{2}(t)\right\rangle$, as given by Eqs. (8) with the appropriate second-order corrections, over the heat bath temperature. This attests that most of the squeezing also occurs inside the heating zone as claimed before. One notices by comparing Figs. 6 and 7 that we obtain very similar level sets for heating and squeezing. This is a consequence of the fact that both of these phenomena have the same cause, as discussed above.

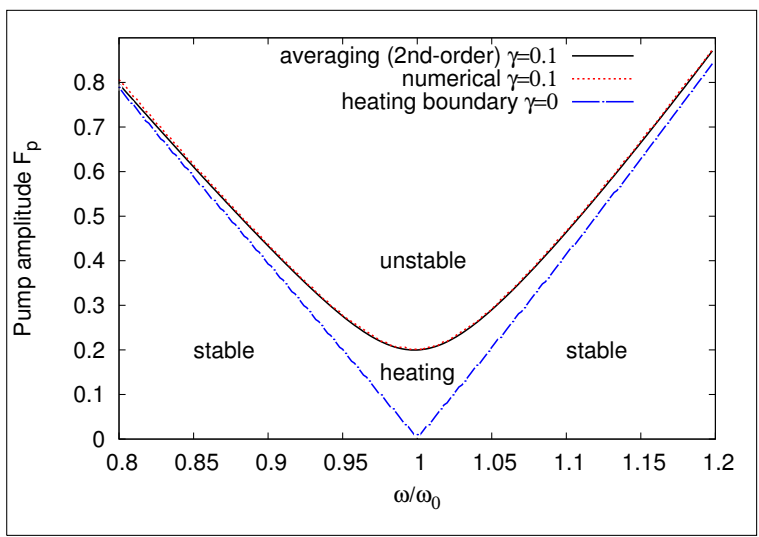

Figure 1: Comparison between numerical and averaging method predictions for the boundary of the first instability zone of the damped parametrically-driven oscillator of Eq. (1). In the region above the thick solid black line lies the unstable zone obtained by numerical computation, the red dashed line is the second-order averaging prediction for the transition line. The heating zone occurs when the Floquet exponents given in Eq. (3) become real. The heating boundary (blue long dashed-dotted line) is equivalent to the transition line to parametric instability when $\gamma=0$.

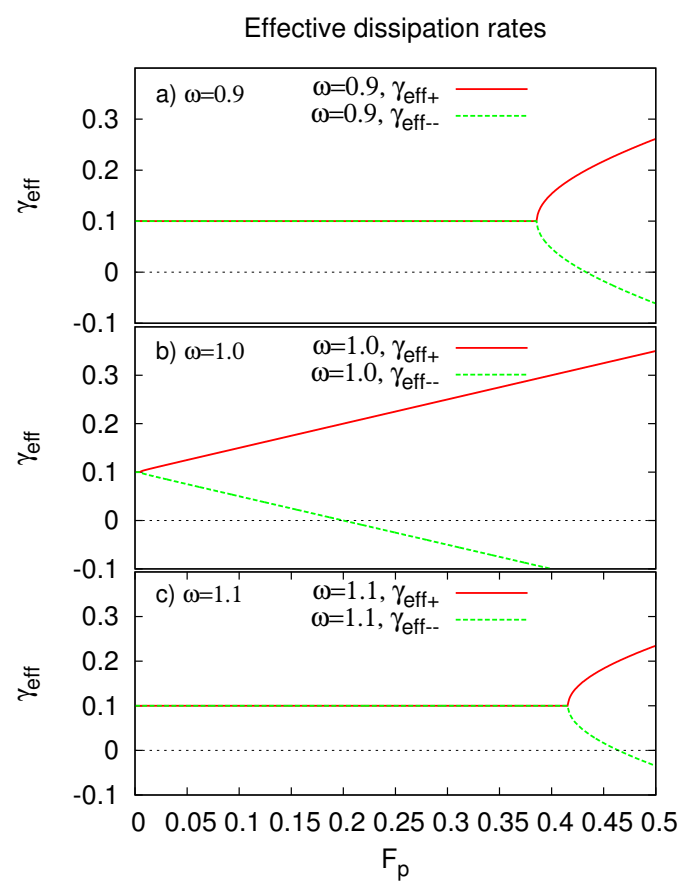

Figure 2: Effective dissipation rates based on second-order averaging approximation obtained from Eq. (3) with $\gamma=0.1$, the other parameters are given in the figure. Heating and squeezing mostly occur when the Floquet multipliers become real and branch off in two different values. Consequently, there is an effective dissipation rate associated with each Floquet multiplier. An effective dissipation rate $\gamma_{\text {eff }}<\gamma$ results in heating of the parametric oscillator. The different values of $\gamma_{\text {eff }}$, one smaller than $\gamma$ and the other larger than $\gamma$, result in squeezing. When $\gamma_{\text {eff- becomes }}$ negative one reaches the transition line to the firstinstability zone of the parametric oscillator.

\section{Conclusion}

Here, we studied a parametrically-driven oscillator with added noise with the objective of understanding what causes heating and thermal squeezing in the stable zone near the transition line of the first parametric instability. We improved on our previous work (Batista, 2011) and obtained a more accurate expression for the Green's functions and, consequently, obtained more precise estimates of the amount of heating and quadrature noise squeezing in the parametrically-driven oscillator. Furthermore, we used an approximate Floquet theory, based on first and second-order averaging approximations, to explain why heating and thermal squeezing occur in the parametricallydriven oscillator with added noise investigated here. These phenomena are related to the onset of real-valued FMs (FMs) with different magnitudes. It was shown that as one FM grows while one gets closer (in parameter space) to the first tran- 
squared Green functions

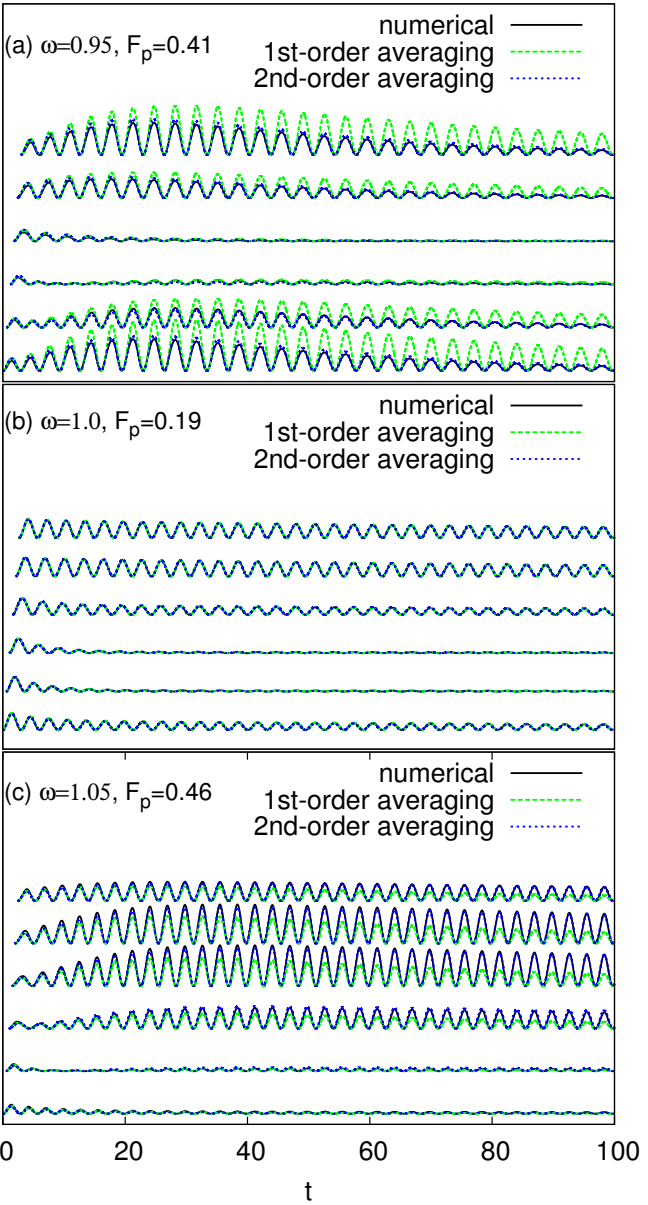

Figure 3: In the frames above we show several squared Green's functions with equally-spaced in time initial conditions in one given period of the parametric driving. They are vertically spaced for clarity, since all asymptotes are zero. In each frame we have a comparison between numerical results given by the numerical integration of Eq. (4) with the initial values $G\left(t, t^{\prime}\right)=0$ and $\frac{\partial}{\partial t} G\left(t, t^{\prime}\right)=1.0$ when $t=t^{\prime}+0^{+}$and the analytical approximate results given by Eqs. (5) or (6).

sition line to instability the other FM decreases. As a consequence, one gets two different effective dissipation rates, while at the same time the input power due to noise remains constant as the pump amplitude is increased. We showed that these effects account for most thermal squeezing and heating observed. We showed as well that the second-order Green's function of the parametric oscillator has the same form as the first-order Green's function, which implies that with a simple parameter change given in the text all first-order results for the amount of heating and thermal squeezing also apply, with increased accuracy, to second-order approximation. This indicates that a Green's function renormalization is very well feasible if one goes to higher orders of approximation

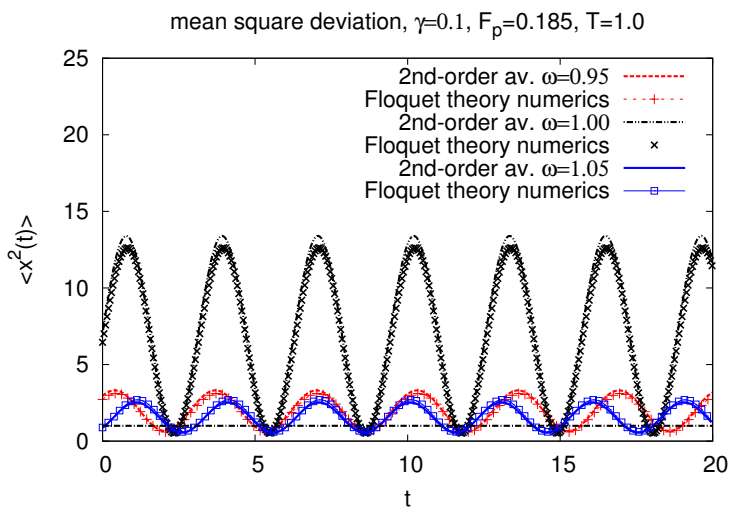

Figure 4: Mean-square deviation $\left\langle x^{2}(t)\right\rangle$ time evolution. Comparison between Floquet theory numerical results and second-order averaging results given by Eq. (8) with the Green's function given by Eq. (6). The dot-dashed line indicates the temperature of the external heat bath. The closer one is to resonance the larger the squeezing amplitude and average dynamical temperature.

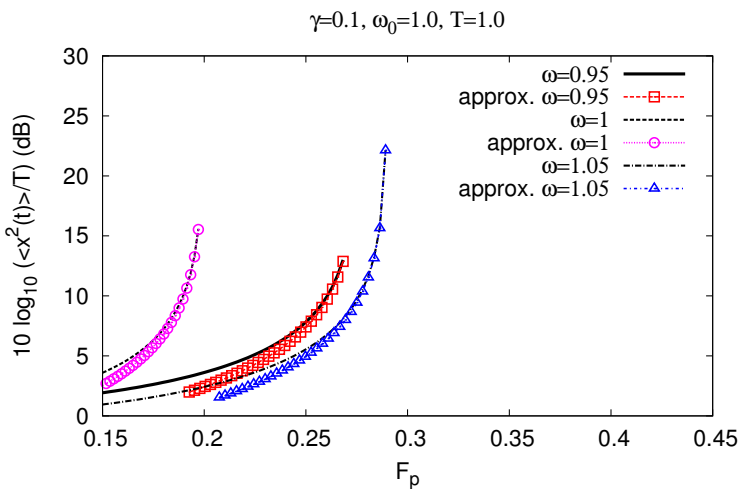

Figure 5: Log plot of the dc component of the mean square displacement in second-order approximation, $\overline{\left\langle x^{2}(t)\right\rangle}$, as given by Eqs. 111). In the parametric oscillator with thermal noise the dynamic temperature of the oscillator grows monotonically until it diverges at the transition line between stable and unstable zones. The simplified approximating curves are given by Eq. (12).

in the averaging method.

The model studied here may be applied for instance to the dynamics of the fundamental mode of an axially loaded doubly-clamped beam resonator. The present model can also be applied to the linear response of ac driven nonlinear oscillators to noise (such as transversally-loaded beam resonators), see for example Ref. (Almog et al., 2007). We note further that this model can be applied as well to the investigation of the dynamics of ions in quadrupole $\mathrm{RF}$ ion guides or traps (Paul, 1990) in the presence of thermal noise. Additionally, the notion that we can have two different effective dissipation rates, one for each 


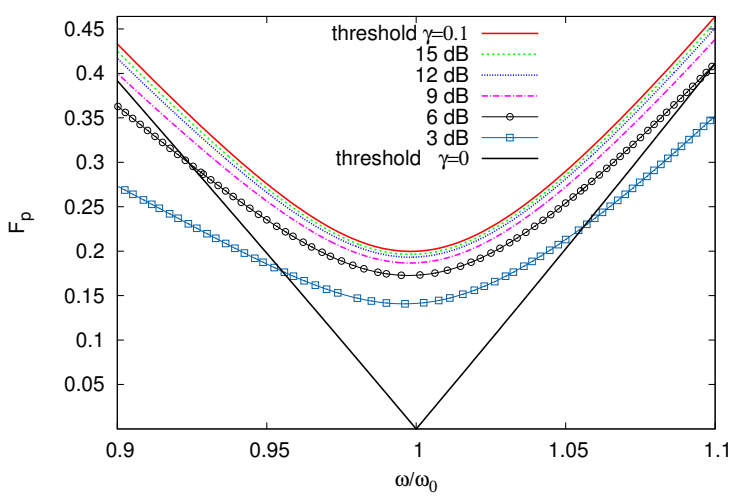

Figure 6: Contour plot of dynamic temperatures (the dc component of the statistical average of the square displacement in second-order approximation, $\overline{\left\langle x^{2}(t)\right\rangle}$, as given by Eqs. (11)) over heat bath temperature in decibel scale. Most of the heating occurs inside the heating zone as predicted by our hypothesis. Each level curve is a dynamic isothermal. The levels are given by the expression $10 \log _{10}\left(\overline{\left\langle x^{2}(t)\right\rangle} / T\right)$.

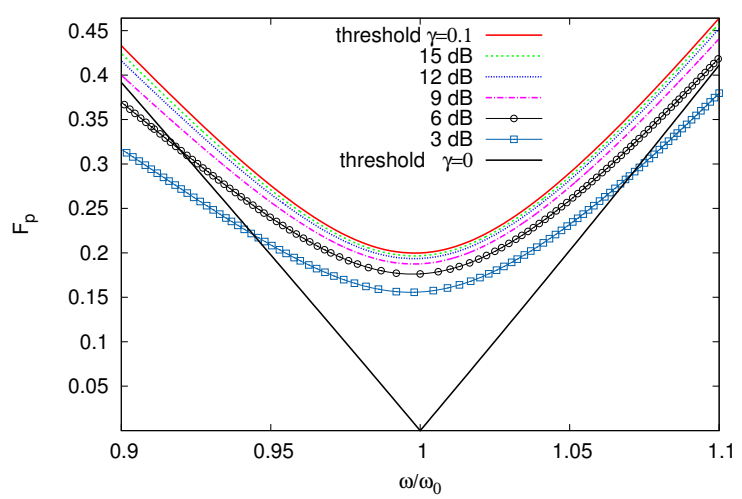

Figure 7: Contour plot of the squeezing amplitude $\left(\sqrt{A_{2 \omega}^{2}+B_{2 \omega}^{2}}\right.$, as given by Eqs. (8) with the appropriate second-order corrections) over heat bath temperature in decibel scale. Most of the squeezing occurs inside the heating zone as predicted by our hypothesis. The levels are given by the expression $10 \log _{10}\left(\sqrt{A_{2 \omega}^{2}+B_{2 \omega}^{2}} / T\right)$.

stable Floquet multiplier, could be used to create control schemes, similar to those of the OGY method (Ott et al., 1990), to reduce the effects of noise even further in parametric amplifiers and in $\mathrm{RF}$ ion traps.

\section{Acknowledgements}

The author would like to thank the financial support of CNPq Grant Universal 14/2012 No. 487416/2012-0.

\section{References}

Almog, R., Zaitsev, S., Shtempluck, O. and Buks, E. (2007). Noise squeezing in a nanomechanical duffing resonator, Phys. Rev. Lett. 98(7): 078103.

DOI: 10.1103/PhysRevLett.98.078103

Batista, A. A. (2011). Cooling, heating, and thermal noise squeezing in a parametrically driven resonator, J. of Stat. Mech. (Theory and Experiment) 2011: P02007. DOI: $10.1088 / 1742-5468 / 2011 / 02 / \mathrm{P} 02007$

Batista, A. A. (2012). Heating and thermal squeezing in parametrically driven oscillators with added noise, Phys. Rev. E 86: 051107. DOI: 10.1103/PhysRevE.86.051107

Batista, A. A. and R. S. N. Moreira (2011). Signalto-noise ratio in parametrically driven oscil-lators, Phys. Rev. E 84(6): 061121. DOI: $10.1103 /$ PhysRevE.84.061121

Guckenheimer, J. and Holmes, P. (1983). Nonlinear Oscillations, Dynamical Systems, and Bifurcations of Vector Fields, SpringerVerlag, New York. DOI: 10.1007/978-1-4612-1140-2

Kubo, R. (1966). The fluctuation-dissipation theorem, Rep. Prog. Phys. 29: 255-284.

DOI: $10.1088 / 0034-4885 / 29 / 1 / 306$

Ott, E., Grebogi, C. and Yorke, J. (1990). Controlling chaos, Phys. Rev. Lett. 64(11): 11961199. DOI: 10.1103/PhysRevLett.64.1196

Paul, W. (1990). Electromagnetic traps for charged and neutral particles, Rev. of Mod. Phys. 62(3): 531-540. DOI: $10.1103 /$ RevModPhys.62.531

Rugar, D. and Grütter, P. (1991). Mechanical parametric amplification and thermomecanical noise squeezing, Phys. Rev. Lett. 67(6): 699. DOI: 10.1103/PhysRevLett.67.699

Verhulst, F. (1996). Nonlinear Differential Equations and Dynamical Systems, Springer-Verlag, New York. DOI: 10.1007/978-3-642-61453-8

Wiesenfeld, K. and McNamara, B. (1985). Perioddoubling systems as small-signal amplifiers, Phys. Rev. Lett. 55: 13. DOI: 10.1103/PhysRevLett.55.13 